\title{
NOTES ON UNIFORM DISTRIBUTION MODULO ONE
}

\author{
G. MYERSON and A. D. POLLINGTON
}

Communicated by J. H. Loxton

\begin{abstract}
We exhibit a sequence $\left(u_{n}\right)$ which is not uniformly distributed modulo one even though for each fixed integer $k \geq 2$ the sequence $\left(k u_{n}\right)$ is u.d. $(\bmod 1)$. Within the set of all such sequences, we characterize those with a well-behaved asymptotic distribution function. We exhibit a sequence $\left(u_{n}\right)$ which is u.d. ( $\left.\bmod 1\right)$ even though no subsequence of the form $\left(u_{k n+j}\right)$ is u.d. $(\bmod 1)$ for any $k \geq 2$. We prove that, if the subsequences $\left(u_{k n}\right)$ are u.d. $(\bmod 1)$ for all squarefree $k$ which are products of primes in a fixed set $\mathscr{P}$, then $\left(u_{n}\right)$ is u.d. $(\bmod 1)$ if the sum of the reciprocals of the primes in $\mathscr{P}$ diverges. We show that this result is the best possible of its type.
\end{abstract}

1980 Mathematics subject classification (Amer. Math. Soc.) (1985 Revision): 11 K 06.

\section{Introduction}

We recall the rudiments of the theory of sequences uniformly distributed modulo one (hereinafter abbreviated as u.d. $(\bmod 1))$. The standard reference for this material is [1].

By $\{x\}$ we mean the fractional part of $x$ (we use the same notation for sets, but context should make the meaning clear). A sequence $\left(u_{n}\right)=$ $\left(u_{1}, u_{2}, \ldots\right)$ of real numbers is said to be u.d. $(\bmod 1)$ if

$$
\lim _{N \rightarrow \infty} \frac{1}{N} \#\left\{n \leq N: \alpha \leq\left\{u_{n}\right\}<\beta\right\}=\beta-\alpha
$$

for all $\alpha, \beta$ with $0 \leq \alpha<\beta \leq 1$. Writing $e(x)$ for $e^{2 \pi i x}$ we can state the Weyl criterion as follows.

This research was supported by the Australian Research Council, grant \#8515178.

(C) 1990 Australian Mathematical Society 0263-6115/90\$A2.00+0.00 
THEOREM 1. The sequence $\left(u_{n}\right)$ is u.d. $(\bmod 1)$ if and only if

$$
\lim _{N \rightarrow \infty} \frac{1}{N} \sum_{n=1}^{N} e\left(h u_{n}\right)=0
$$

for all non-zero integers $h$.

We conclude our review with some immediate consequences of the Weyl criterion.

Corollary 1. If $\left(u_{n}\right)$ is u.d. $(\bmod 1)$ then so is $\left(k u_{n}\right)$ for any non-zero integer $k$.

Corollary 2. If for fixed $k \geq 1$ and for all $j, 1 \leq j \leq k$, the sequence $\left(u_{k n+j}\right)$ is u.d. $(\bmod 1)$ then $\left(u_{n}\right)$ is u.d. $(\bmod 1)$.

\section{Multiples}

In this section we first show by example that even a very weak converse of Corollary 1 is false.

Theorem 2. There exists a sequence $\left(u_{n}\right)$, not $u . d$. (mod 1), such that $\left(k u_{n}\right)$ is u.d. $(\bmod 1)$ for all integers $k \geq 2$.

Proof. Let $g(x)=x+\frac{1}{2 \pi} \sin 2 \pi x$. Note that $g$ is a continuous, increasing function on $[0,1], g(0)=0$, and $g(1)=1$. Thus $g$ has an inverse, $h$, with these same properties. Let $\left(x_{n}\right)$ be any sequence u.d. $(\bmod 1)$, and let $u_{n}=h\left(\left\{x_{n}\right\}\right), n=1,2, \ldots$. We claim that $\left(u_{n}\right)$ satisfies the conditions of the theorem.

For any sequence $\left(v_{n}\right)$, and for $0 \leq \alpha<\beta \leq 1$, let us write $\operatorname{pr}(\alpha \leq v<\beta)$ for

$$
\lim _{N \rightarrow \infty} \frac{1}{N} \#\left\{n \leq N: \alpha \leq v_{n}<\beta\right\},
$$

if the limit exists. Then

$$
\begin{aligned}
\operatorname{pr}(\alpha \leq u<\beta) & =\operatorname{pr}(\alpha \leq h(\{x\})<\beta) \\
& =\operatorname{pr}(g(\alpha) \leq\{x\}<g(\beta))=g(\beta)-g(\alpha)
\end{aligned}
$$

since $x_{n}$ is u.d. $(\bmod 1)$. But $g(\beta)-g(\alpha)=\beta-\alpha+\frac{1}{2 \pi}(\sin 2 \pi \beta-\sin 2 \pi \alpha)$ which is, in general, not equal to $\beta-\alpha$, so $\left(u_{n}\right)$ is not u.d. $(\bmod 1)$. 
Now let $k$ be any integer greater than 1 . Then

$$
\begin{aligned}
\operatorname{pr}(\alpha \leq\{k u\}<\beta) & =\operatorname{pr}(\alpha \leq\{k h(x)\}<\beta) \\
& =\sum_{r=0}^{k-1} \operatorname{pr}(\alpha+r \leq k h(x)<\beta+r) \\
& =\sum_{r=0}^{k-1} \operatorname{pr}\left(\frac{\alpha+r}{k} \leq h(x)<\frac{\beta+r}{k}\right) \\
& =\sum_{r=0}^{k-1} \operatorname{pr}\left(g\left(\frac{\alpha+r}{k}\right) \leq x<g\left(\frac{\beta+r}{k}\right)\right) \\
& =\sum_{r}\left(g\left(\frac{\beta+r}{k}\right)-g\left(\frac{\alpha+r}{k}\right)\right) \\
& =\beta-\alpha+\frac{1}{2 \pi} \sum_{r}\left(\sin 2 \pi\left(\frac{\beta+r}{k}\right)-\sin 2 \pi\left(\frac{\alpha+r}{k}\right)\right) \\
& =\beta-\alpha
\end{aligned}
$$

since $\sum_{r=0}^{k-1} \sin 2 \pi(z+r / k)=0$ for all real $z$ and $k=2,3, \ldots$.

We now show that all the "nice" examples of sequences with the property given in Theorem 2 are essentially those produced in the proof of that theorem.

TheOREM 3. Suppose $\left(k u_{n}\right)$ is u.d. $(\bmod 1)$ for $k=2,3, \ldots$, and $g(x)=\operatorname{pr}(0 \leq\{u\}<x)$ exists and is continuous. Then

$$
g(x)=x+c_{1}(1-\cos 2 \pi x)+c_{2} \sin 2 \pi x
$$

for some constants $c_{1}, c_{2}$, and, if $x_{n}=g\left(u_{n}\right)$, then $\left(x_{n}\right)$ is u.d. $(\bmod 1)$.

REMARK. In an earlier version of this paper the conclusion of this theorem rested on the stronger hypothesis that $g$ be differentiable. We thank Boping Jin for showing us how to weaken the hypothesis.

Proof. By hypothesis we have, for $k=2,3, \ldots$, and $0 \leq \alpha<\beta \leq 1$,

$$
\begin{aligned}
\beta-\alpha & =\operatorname{pr}(\alpha \leq\{k u\}<\beta) \\
& =\sum_{r=0}^{k-1} \operatorname{pr}(\alpha+r \leq k\{u\}<\beta+r) \\
& =\sum_{r=0}^{k-1} \operatorname{pr}\left(\frac{\alpha+r}{k} \leq\{u\}<\frac{\beta+r}{k}\right) \\
& =\sum_{r}\left(g\left(\frac{\beta+r}{k}\right)-g\left(\frac{\alpha+r}{k}\right)\right) .
\end{aligned}
$$


Let $\alpha=0, \beta=x$; then $\sum_{r} g((x+r) / k)-x=\sum_{r} g(r / k)$, a constant. Thus for $m \geq 1$ we have

$$
\int_{0}^{1} e(-m x) \sum_{r} g\left(\frac{x+r}{k}\right) d x=\int_{0}^{1} x e(-m x) d x .
$$

The right side of this equation is simply $-i / 2 \pi m$. Call the left side $a_{m}$; we get

$$
\begin{aligned}
a_{m} & =\sum_{r=0}^{k-1} \int_{0}^{1} e(-m x) g\left(\frac{x+r}{k}\right) d x \\
& =\sum_{r} k \int_{r / k}^{(r+1) / k} e(-m(k y-r)) g(y) d y \\
& =k \int_{0}^{1} e(-m k y) g(y) d y .
\end{aligned}
$$

Thus,

$$
\int_{0}^{1} e(-n y) g(y) d y=-\frac{i}{2 \pi n} \quad \text { for } n=k, 2 k, \ldots
$$

But $k=2,3, \ldots$, so

$$
\int_{0}^{1} e(-n y) g(y) d y=-\frac{i}{2 \pi n} \quad \text { for } n=2,3, \ldots
$$

Thus, the Fourier coefficients of $g(x)$ and of $x$ are identical for $n \geq 2$, so

$$
g(x)=x+c_{1}+c_{2} \sin 2 \pi x+c_{3} \cos 2 \pi x
$$

for some constants $c_{1}, c_{2}, c_{3}$. Since $g(0)=0$ we have $c_{1}+c_{3}=0$, and we have established the form of $g$. Then

Now let $x_{n}=g\left(u_{n}\right)$. Note that $g$ is increasing, so $h=g^{-1}$ is defined.

$$
\begin{aligned}
\operatorname{pr}(\alpha \leq x<\beta) & =\operatorname{pr}(\alpha \leq g(u)<\beta) \\
& =\operatorname{pr}(h(\alpha) \leq u<h(\beta)) \\
& =g(h(\beta))-g(h(\alpha))=\beta-\alpha,
\end{aligned}
$$

so $\left(x_{n}\right)$ is u.d. $(\bmod 1)$.

\section{Subsequences}

In this section we first show by example that even a very weak converse of Corollary 2 is false. We use $p$ only for primes, we write $n(\bmod p)$ for the least non-negative residue of $n$ modulo $p$, and we write $P(M)$ for $\prod_{p \leq M} p$. 
THEOREM 4. The sequence $\left(u_{n}\right)$ given by

$$
u_{n}=\sum_{p}^{\infty} \frac{n(\bmod p)}{P(p)},
$$

is u.d. $(\bmod 1)$, but for fixed $k, j, k \geq 2$, no subsequence of the form $u_{k n+j}$ is u.d. $(\bmod 1)$.

Our proof uses some simple facts about the Cantor expansion of a real number. We collect these facts in a lemma.

Lemma. Every $\alpha$ in $[0,1)$ has an expansion of the form

$$
\alpha=\sum_{p}^{\infty} \frac{\alpha_{p}}{P(p)}, \quad \text { where } \alpha_{p} \text { are integers, } 0 \leq \alpha_{p} \leq p-1 \text {. }
$$

If we exclude expansions in which $\alpha_{p}=p-1$ for all $p$ sufficiently large, the expansion is unique. The expansion of $\alpha$ terminates (that is, $\alpha_{p}=0$ for all $p$ sufficiently large) if and only if $\alpha=c / P(M)$ for some $M$ and some integer $c, 0 \leq c<P(M)$. Let

$$
\frac{c}{P(M)}=\sum_{p \leq M} \frac{\beta_{p}}{P(p)}, \quad 0 \leq \beta_{p} \leq p-1 .
$$

If $p$ is the largest prime not exceeding $M$, then $c \equiv \beta_{p}(\bmod p)$; if

$$
\frac{c}{P(M)} \leq \alpha<\frac{c+1}{P(M)}
$$

then $\alpha_{p}=\beta_{p}$ for $p \leq M$.

Proof of Theorem. We first prove that $\left(u_{n}\right)$ is u.d. $(\bmod 1)$. Given $\alpha$ and $\beta$ with $0 \leq \alpha<\beta \leq 1$, and given $\varepsilon>0$, let $M$ be such that $P(M)>\varepsilon^{-1}$, let $a=[\alpha P(M)]$, and let $b=[\beta P(M)]$. Then

$$
\begin{aligned}
\#\left\{n \leq N: \alpha \leq u_{n}<\beta\right\} & =\#\left\{n \leq N: \frac{a}{P(M)} \leq u_{n}<\frac{b+1}{P(M)}\right\} \\
& =\sum_{c=a}^{b} \#\left\{n \leq N: \frac{c}{P(M)} \leq u_{n}<\frac{c+1}{P(M)}\right\} \\
& =\sum_{c=a}^{b} \#\left\{n \leq N: n \equiv \beta_{p}(c)(\bmod p), p \leq M\right\} \\
& \leq \sum_{c=a}^{b}\left(\frac{N}{P(M)}+1\right) \\
& =(b-a+1) \frac{N}{P(M)}+b-a+1,
\end{aligned}
$$


where

$$
\frac{c}{P(M)}=\sum_{p \leq M} \frac{\beta_{p}(c)}{P(p)}
$$

Thus

$$
\frac{1}{N} \#\left\{n \leq N: \alpha \leq u_{n}<\beta\right\} \leq \beta-\alpha+3 \varepsilon
$$

for $N$ sufficiently large. A similar argument shows that

$$
\frac{1}{N} \#\left\{n \leq N: \alpha \leq u_{n}<\beta\right\} \geq \beta-\alpha-3 \varepsilon
$$

for $N$ sufficiently large, whence $\left(u_{n}\right)$ is u.d. $(\bmod 1)$.

Now consider $\left(u_{k n+j}\right), k$ and $j$ fixed, $k \geq 2, n=1,2, \ldots$ Let $p$ be any prime dividing $k$, so $\left(u_{k n+j}\right)$ is a subsequence of $\left(u_{p n+j}\right)$. Let $j^{\prime}$ be any integer with $j^{\prime} \not \equiv j(\bmod p)$, and $0 \leq j^{\prime}<P(p)$. Then

$$
\frac{j^{\prime}}{P(p)} \leq u_{p n+j}<\frac{j^{\prime}+1}{P(p)}
$$

is impossible, so

$$
\frac{j^{\prime}}{P(p)} \leq u_{k n+j}<\frac{j^{\prime}+1}{P(p)}
$$

is impossible, and $\left(u_{k n+j}\right)$ is not u.d. $(\bmod 1)$.

Our final result can be seen as complementary to Corollary 2 .

THEOREM 5. Let $\mathscr{P}$ be a set of primes such that $\sum_{p \in \mathscr{P}} \frac{1}{p}$ diverges. Let $\mathscr{K}$ denote the set of squarefree integers divisible only by primes in $\mathscr{P}$. If $\left(u_{k n}\right)$ is u.d. $(\bmod 1)$ for every $k>1$ in $\mathscr{K}$ then $\left(u_{n}\right)$ is u.d. $(\bmod 1)$.

RemarK. If $\mathscr{P}$ is a set of primes such that $\sum_{p \in \mathscr{P}} \frac{1}{p}$ converges then given any irrational $\alpha$ the sequence $\left(u_{n}\right)$ given by

$$
u_{n}= \begin{cases}n \alpha, & \text { if } p \mid n \text { for some } p \in \mathscr{P}, \\ 0, & \text { otherwise }\end{cases}
$$

has the property that $\left(u_{k n}\right)$ is u.d. $(\bmod 1)$ for any $k$ divisible by some prime in $\mathscr{P}$, but $\left(u_{n}\right)$ is not u.d. $(\bmod 1)$ since $u_{n}=0$ on a set of positive density.

Proof. We put

$$
P(\mathscr{P}, M)=\prod_{\substack{p \in \mathscr{P} \\ p \leq M}} p
$$


By the Weyl criterion, we have

$$
\lim _{N \rightarrow \infty} \frac{1}{N} \sum_{n=1}^{N} e\left(m u_{k n}\right)=0
$$

for all non-zero integers $m$ and all $k \in \mathscr{K}, k>1$. Thus given $M>0$ there is an $N_{0}=N_{0}(m, M)$ such that if $N>N_{0}$ then

$$
\frac{k}{N}\left|\sum_{n=1}^{N / k} e\left(m u_{k n}\right)\right|<\frac{1}{M}
$$

for all $k \mid P(\mathscr{P}, M), k>1$. Now

$$
\begin{aligned}
\frac{1}{N}\left|\sum_{n=1}^{N} e\left(m u_{n}\right)\right| & \leq \frac{1}{N} \sum_{\substack{k \mid P(\mathscr{P}, M) \\
k>1}}|\mu(k)|\left|\sum_{n=1}^{N / k} e\left(m u_{k n}\right)\right|+\frac{1}{N} \sum_{\substack{n \leq N \\
(n, P(\mathscr{P}, M))=1}} 1 \\
& \leq \frac{1}{M} \prod_{p \mid P(\mathscr{P}, M)}\left(1+\frac{1}{p}\right)+\prod_{p \mid P(\mathscr{P}, M)}\left(1-\frac{1}{p}\right)
\end{aligned}
$$

by (1). The first term on the right goes to 0 as $M$ goes to infinity:

$$
\begin{aligned}
\prod_{p \mid P(\mathscr{P}, M)}\left(1+\frac{1}{p}\right) & \leq \prod_{p \leq M}\left(1+\frac{1}{p}\right) \\
& =\exp \sum_{p \leq M} \log \left(1+\frac{1}{p}\right) \leq \exp \sum_{p \leq M} \frac{1}{p}=O(\log M) .
\end{aligned}
$$

Since $\sum_{p \in \mathscr{P}} \frac{1}{p}$ diverges, the second term on the right also goes to zero as $M$ goes to infinity. Hence, $\left(u_{n}\right)$ is u.d. $(\bmod 1)$, by Weyl's criterion.

\section{Multiples in higher dimensions}

We conclude with a discussion of uniform distribution in higher dimensions, where the statement analogous to Theorem 2 goes badly wrong. A sequence $\left(u_{n}\right)$ of real $m$-tuples is said to be u.d. $(\bmod 1)$ if

$$
\lim _{N \rightarrow \infty} \frac{1}{N} \#\left\{n \leq N: \boldsymbol{\alpha} \leq \mathbf{u}_{n}<\boldsymbol{\beta}\right\}=|\boldsymbol{\beta}-\boldsymbol{\alpha}|
$$

for all $\boldsymbol{\alpha}, \boldsymbol{\beta}$ with $\mathbf{0} \leq \boldsymbol{\alpha}<\boldsymbol{\beta} \leq \mathbf{1}$; here, and below, $\left(\mathbf{u}_{n}\right)$ means $\left(\left\{u_{n}^{(1)}\right\}, \ldots\right.$, $\left.\left\{u_{n}^{(m)}\right\}\right) ;\left(x_{1}, \ldots, x_{m}\right)<\left(y_{1}, \ldots, y_{m}\right)$ means $x_{j}<y_{j}$ for $j=1, \ldots, m$; $\left|\left(x_{1}, \ldots, x_{m}\right)\right|$ means $x_{1} x_{2} \cdots x_{m} ; 0$ means $(0, \ldots, 0)$, and 1 means $(1, \ldots, 1)$. The Weyl criterion is 
THEOREM $1^{\prime}$. The sequence $\left(\mathbf{u}_{n}\right)$ is $u . d .(\bmod 1)$ if and only if

$$
\lim _{N \rightarrow \infty} \frac{1}{N} \sum_{n=1}^{N} e\left(\mathbf{h} \cdot \mathbf{u}_{n}\right)=0
$$

for all non-zero integer $m$-tuples $\mathbf{h}$.

An immediate consequence is

COROLlaRY $1^{\prime}$. If $\left(\mathbf{u}_{n}\right)$ is u.d. (mod 1$)$ then so is $\left(A \mathbf{u}_{n}\right)$ for any nonsingular integer matrix $A$.

Another simple consequence is

Corollary 3. If $A$ is an integer matrix with determinant \pm 1 and $\left(A \mathbf{u}_{n}\right)$ is u.d. $(\bmod 1)$ then $\left(\mathbf{u}_{n}\right)$ is u.d. $(\bmod 1)$.

Proof. Under the hypotheses, $A^{-1}$ has integer entries, so, by the previous corollary, $\left(A^{-1} A \mathbf{u}_{n}\right)$ is u.d. $(\bmod 1)$.

A statement analogous to Theorem 2 would be, "there exists a sequence $\left(\mathbf{u}_{n}\right)$, not u.d. ( $\left.\bmod 1\right)$, such that $\left(A \mathbf{u}_{n}\right)$ is u.d. (mod 1$)$ for all integer matrices $A$ with $\operatorname{det} A \geq 2$." However, this statement is far from being true. Instead we have

THEOREM 6. Let $S$ be a set of $m \times m$ integer matrices, and suppose that for every integer row $m$-vector $\mathbf{h}$ there exists a matrix $A$ in $S$ and an integer row $m$-vector $\mathbf{k}$ such that $\mathbf{k} A=\mathbf{h}$. Then if $\left(A \mathbf{u}_{n}\right)$ is u.d. $(\bmod 1)$ for all $A$ in $S$, then $\left(\mathbf{u}_{n}\right)$ is u.d. $(\bmod 1)$.

Proof. Given a non-zero integer $m$-tuple $\mathbf{h}$, considered as a row vector, let $A$ in $S$ and $\mathbf{k}$ an integer row-vector be such that $\mathbf{k} A=\mathbf{h}$. Then

$$
\sum_{n=1}^{N} e\left(\mathbf{h} \cdot \mathbf{u}_{n}\right)=\sum_{n} e\left(\mathbf{k} A \cdot \mathbf{u}_{n}\right)=\sum_{n} e\left(\mathbf{k} \cdot A \mathbf{u}_{n}\right)=o(N),
$$

since $\left(A u_{n}\right)$ is u.d. $(\bmod 1)$. Thus, $\left(\mathbf{u}_{n}\right)$ is u.d. $(\bmod 1)$.

EXAMPLE. Let $m=2$. One easily verifies that

$$
S=\left\{\left(\begin{array}{ll}
2 & 0 \\
0 & 1
\end{array}\right),\left(\begin{array}{ll}
1 & 0 \\
0 & 2
\end{array}\right),\left(\begin{array}{cc}
1 & -1 \\
1 & 1
\end{array}\right)\right\}
$$

has the property required. If $c$ is even, then $\left(\begin{array}{ll}c & d\end{array}\right)=\left(\begin{array}{ll}c / 2 & d\end{array}\right)\left(\begin{array}{ll}2 & 0 \\ 0 & 1\end{array}\right)$; if $d$ is even, then $\left(\begin{array}{ll}c & d\end{array}\right)=\left(\begin{array}{ll}c & d / 2\end{array}\right)\left(\begin{array}{ll}1 & 0 \\ 0 & 2\end{array}\right)$; if $c$ and $d$ are both odd (or both even), 
then $(c d)=((c-d) / 2(c+d) / 2)\left(\begin{array}{cc}1 & -1 \\ 1 & 1\end{array}\right)$. Thus if $\left(A u_{n}\right)$ is u.d. $(\bmod 1)$ for all $A$ in $S$, then $\left(\mathbf{u}_{n}\right)$ is u.d. $(\bmod 1)$.

\section{Note added in proof}

Peter Sarnak has pointed out that Theorem 3 holds under the weaker hypothesis that $g(x)$ exists as a measure. Also, Michel Mendès France has pointed out to us that Theorem 2 is a special case of the main theorem of $F$. Dress and M. Mendès France, 'Caractérisation des ensembles normaux dans Z,' Acta Arith. 17 (1970), 115-120.

\section{References}

[1] L. Kuipers and H. Niederreiter, Uniform distribution of sequences, (Wiley, 1974).

School of Mathematics, Physics, Computing and Electronics Macquarie University Australia 2109
Department of Mathematics Brigham Young University Provo, Utah 84602 U.S.A. 